\title{
Estrategias de modelado patrimonial en HBIM, aplicación a la lectura estratigráfica del muro de fachada del cuadrante renacentista de la catedral de Sevilla*
}

\section{Heritage modelling strategies in HBIM, application to the stratigraphic reading of the façade of the Renaissance quadrant of the Cathedral of Seville}

\author{
Roque Angulo-Fornos ${ }^{1}$, Manuel Castellano-Román², Francisco Pinto-Puerto ${ }^{3}$ \\ Departamento de Expresión Gráfica Arquitectónica. Universidad de Sevilla
}

\begin{abstract}
RESUMEN
Este artículo presenta diferentes estrategias de modelado digital del proceso constructivo de un elemento arquitectónico en un entorno HBIM, analizando los Niveles de Conocimiento -LOK- que permiten cada una de ellas. Partiendo de la captura digital fotogramétrica de uno de los muros de la fachada de las dependencias capitulares de la catedral de Sevilla, se ha generado un modelo conceptual inicial orientado a la definición genérica del bien y a su encuadre dentro del conjunto catedralicio. Posteriormente, con objeto de experimentar la potencialidad de aplicación de estas herramientas al análisis arqueológico y arquitectónico, se han transportado sus resultados a este entorno HBIM, alcanzando la caracterización tridimensional de cada una de sus unidades constructivas básicas de la fachada a diversos niveles, desde unidades estratigráficas formadas por regiones hasta las unidades materiales de cada sillar. Por lo tanto, la lectura estratigráfica no se limita al análisis de una superficie de un paramento aislado, sino que se aborda desde su realidad tridimensional general, la catedral. El alto nivel de conocimiento alcanzado en este modelo más desarrollado ha permitido utilizarlo como base para el registro y gestión de la información patrimonial, permitiendo visualizaciones temáticas gráficas y alfanuméricas.
\end{abstract}

Palabras clave: HBIM; análisis estratigráfico; patrimonio arquitectónico; catedral de Sevilla.

\section{ABSTRACT}

This paper discusses different heritage modelling strategies included in the HBIM model, analyzing the Levels of Knowledge -LOK- allowed by each of them. Starting with the photogrammetric digital capture of the Renaissance Quadrant façade of the Cathedral of Seville, an initial conceptual model has been generated, oriented towards the generic definition of the asset and its location in the context of cathedral ensemble. The archaeological and architectonical analysis of the constructive process have enhanced the knowledge of the study case and, therefore, the possibility to increase the level of development of the HBIM model, which have reached the three-dimensional characterization of every single basic constructive unit of the façade. Therefore, the stratigraphic analysis is not limited to the surface of the wall, but characterized as a three-dimensional reality, approaching thus the cathedral. The high level of knowledge reached by this model has made it possible to take it as basis for registering and managing heritage information, allowing themed graphic and alphanumeric views.

Key words: HBIM; stratigraphic analysis; architectural heritage; Cathedral of Seville.

Recibido: 03-02-2020. Aceptado: 19-03-2020. Publicado: 13-04-2021

\footnotetext{
Este trabajo forma parte de las acciones del proyecto HAR2016-78113-R del Plan Nacional de I+D+i del Ministerio de Economía y Competitividad. roqueaf@us.es / ORCID iD: https://orcid.org/0000-0002-0401-8377

manuelcr@us.es / ORCID iD: https://orcid.org/0000-0003-4787-447X

fspp@us.es / ORCID iD: https://orcid.org/0000-0003-4608-6818
} 
Cómo citar este artículo / Citation

Angulo-Fornos, R., Castellano-Román, M. y Pinto-Puerto, F. 2021: "Estrategias de modelado patrimonial en HBIM, aplicación a la lectura estratigráfica del muro de fachada del cuadrante renacentista de la catedral de Sevilla", Arqueología de la Arquitectura, 18: e109. https://doi. org/10.3989/arq.arqt.2021.001

Copyright: (C) CSIC, 2021. (C) UPV/EHU Press, 2021. Este es un artículo de acceso abierto distribuido bajo los términos de la licencia de uso y distribución Creative Commons Reconocimiento 4.0 Internacional (CC BY 4.0).

\section{INTRODUCCIÓN}

En los últimos años se ha producido un amplio repertorio de aportaciones en relación con el uso patrimonial de la metodología BIM, agrupadas bajo la denominación HBIM pero muy diferentes en cuanto a su planteamiento conceptual, estrategias metodológicas y resultados esperados. A grandes rasgos, pueden reconocerse experiencias orientadas al modelado de complejas geometrías paramétricas para la reconstrucción virtual de arquitecturas desaparecidas, experiencias centradas en la obtención automática o semiautomática de modelos de alta precisión geométrica a partir de capturas digitales de edificios patrimoniales existentes y, finalmente, otros casos donde el modelo HBIM forma parte de un proceso de levantamiento y análisis arquitectónico cuyas características evolucionan según el nivel de conocimiento del edificio (Rua y Gil 2014).

La investigación que se presenta se sitúa dentro de este último grupo, poniendo el foco en la capacidad de los modelos HBIM para el registro de las diferentes fases constructivas reconocibles en una lectura estratigráfica de los paramentos de una estructura arquitectónica emergente. Se presentan diferentes estrategias de modelado patrimonial, integradas en el mismo modelo HBIM, que permiten presentar diferentes Niveles de Conocimiento, LOK.

Estos LOK son una traslación del concepto BIM del Nivel de Desarrollo - LOD- al ámbito del patrimonio arquitectónico. El Nivel de Conocimiento LOK100 está asociado a la identificación del bien patrimonial y a la investigación y generación de conocimiento sobre el mismo. El LOK200 permite una caracterización gráfica y de información suficiente para el desarrollo de las acciones relacionadas con la protección jurídica del bien y su planificación estratégica o plan director. El LOK300 avanza en la caracterización de las entidades gráficas hasta el punto de poder mostrar los resultados de investigaciones especializadas realizadas con metodología arqueológica u otros estudios disciplinares específicos de auscultación y diagnóstico. El LOK400 contempla las acciones específicas de conservación e intervención sobre los elemen- tos del inmueble. Finalmente, el LOK500 se alcanza en aquellos modelos HBIM que resulten operativos para la gestión eficiente e integral de la tutela patrimonial (Castellano-Román y Pinto-Puerto 2019).

Sin renunciar a la precisión geométrica que permite la captura digital fotogramétrica, se ha generado un modelo conceptual inicial orientado a la definición genérica del bien y a su encuadre dentro del conjunto arquitectónico. El análisis arqueológico y arquitectónico desarrollado sobre el proceso constructivo (Pinto-Puerto 2013) ha permitido el avance en el conocimiento del caso de estudio y, en consecuencia, la posibilidad de aumentar el nivel de desarrollo del modelo HBIM, que ha alcanzado la caracterización tridimensional de cada una de las unidades constructivas básicas, que se ha llevado al límite alcanzando en este caso la discretización en sillares. Por lo tanto, la lectura estratigráfica no se limita al análisis de la superficie del paramento, sino que se aborda desde su realidad tridimensional.

El alto nivel de conocimiento alcanzado en el modelo ha permitido utilizarlo como base para el registro y gestión de la información patrimonial, permitiendo visualizaciones temáticas gráficas y alfanuméricas que favorecen su conocimiento y dan soporte, además, a otras acciones de la tutela patrimonial como la difusión o la conservación preventiva.

Para mostrar de una manera sintética los resultados de este trabajo, ajustándonos a la extensión de la aportación, se ha concretado la experiencia a una parte del edificio tratado, con suficiente autonomía formal y constructiva, el muro suroeste del cuadrante renacentista de la catedral de Sevilla.

Este conjunto de construcciones y espacios, de diverso origen y evolución, se encuentran adosados al ángulo suroriental del templo, confinados por un imponente muro de fachada, desplegado entre la Puerta de San Cristóbal y la Puerta de Campanillas, que le confiere la apariencia de un todo unitario y continuo, solo alterado por la disposición de algunos huecos y por la variación del orden en su extremo oriental (Fig. 1).

La fachada del cuadrante renacentista encierra edificaciones como la Sacristía de los Cálices, el Patio de los 

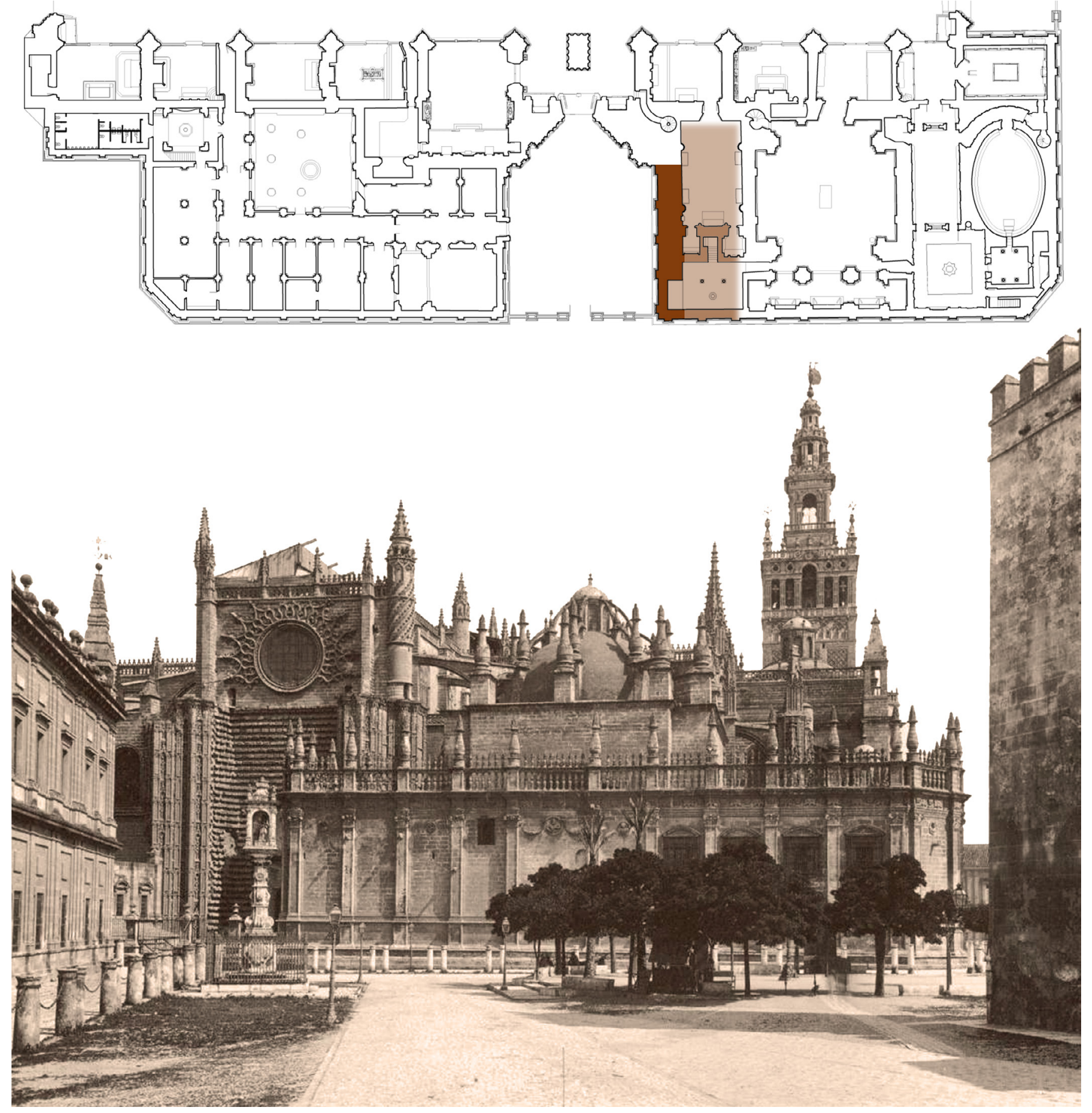

Figura 1. Situación en planta y fotografía de archivo del caso de estudio.

Óleos, la Sacristía Mayor, el Antecabildo, el Patio del Mariscal, la Sala Capitular, la escalera y el cuarto de las trazas. Cada una de ellas contiene elementos arquitectónicos, configuraciones espaciales y procesos constructivos bien diferenciados, trazados y dirigidos por los más importantes maestros mayores del arzobispado que protagonizaron el tránsito a la Edad Moderna y el desarrollo pleno de un lenguaje renacentista: Gil de Hontañón, Alonso Rodríguez, Diego de Riaño, Martín de Gainza, Hernán Ruiz o Asencio de Maeda, entre otros. A su vez, 
estas construcciones fueron amortizando progresivamente las antiguas estructuras defensivas que separaban la mezquita, y posteriormente la catedral gótica, del alcázar (Arévalo-Rodríguez 2011).

El proceso constructivo del cuadrante renacentista puede acotarse entre 1528 y 1596, año en que se levantaron las contadurías. El autor de la traza inicial de este conjunto fue el maestro Diego de Riaño, y su construcción se prolonga tras su muerte hasta los años centrales del siglo XVI en los que se terminan de los remates superiores. La fábrica se inició por el extremo oeste, justo por el muro que tratamos, tras demoler las edificaciones previas, levantar las cimentaciones del conjunto y nivelar el recinto (Tabales-Rodríguez y Jiménez Sancho 2002) aprovechando parte de los muros de la sacristía que comenzó a levantar el maestro Alonso Rodríguez tras cerrar el cimborrio en 1506, inconclusa tras la desgraciada caída de esa estructura en 1511.

Por lo tanto, este edificio se inicia de una forma peculiar: levantando el muro de fachada que delimita el cuadrante renacentista antes que las propias dependencias, dando así prioridad a la protección del recinto. Esta forma de proceder es recurrente en el proceso constructivo de la propia catedral, en la que, tras eliminar un elemento de protección perimetral, se construía inmediatamente otro nuevo dentro del cual se desarrollaban fases constructivas posteriores (Pinto-Puerto 2006, 2019: 229). La fachada se compone con un orden corintio apilastrado sobre un potente basamento continuo que recorre toda la longitud de la fachada y sirve para asumir el desnivel del terreno colindante. Sobre estas pilastras se desarrolla un entablamento completo formado por arquitrabe, friso decorado con cartelas y una potente cornisa. El conjunto queda rematado por una balaustrada modulada entre pináculos entorchados situados sobre las pilastras, que se duplican en las esquinas. Toda la fábrica es de sillares de piedra de dos orígenes litológicos bien diferenciadas: las pilastras y entablamento es de una piedra calcarenita más compacta procedente de las canteras de Utrera, que permite una labra más fina y detallada, mientras los fondos lisos entre éstas se han fabricado con piedra más porosa procedente de la Sierra de San Cristóbal (Rodríguez Estévez 1998).

El caso de estudio se limita al tramo de fachada que comienza a levantarse adosado al estribo sureste del crucero de la catedral y continua, demoliendo parte del muro almohade de separación con el Alcázar, adosada a los muros de la principiada sacristía de los Cálices. El proceso puede observarse a nivel de volúmenes conceptuales en la secuencia de la Figura 2.

Este muro, de 1,10 m de espesor medio, fue construyéndose en diversos impulsos constructivos irregulares entre 1528 y 1543 según se desprende de la lectura estratigráfica de sus paramentos (Pinto-Puerto 2013). Esta lectura revela un proceso constructivo sin grandes interrupciones, pero sujeto a algunos cambios de criterio. Las diferentes unidades constructivas no responden a alteraciones de épocas posteriores, sino a los condicionantes del propio proceso de ejecución de la obra de Riaño, sugiriendo cuestiones relacionadas con la permanencia de la sacristía anterior, el adosamiento obligado a la fábrica gótica previa, la introducción de nuevas canteras en el suministro de material pétreo, o los reajustes del proyecto renacentista para las estancias capitulares sobre el proyecto gótico anterior.
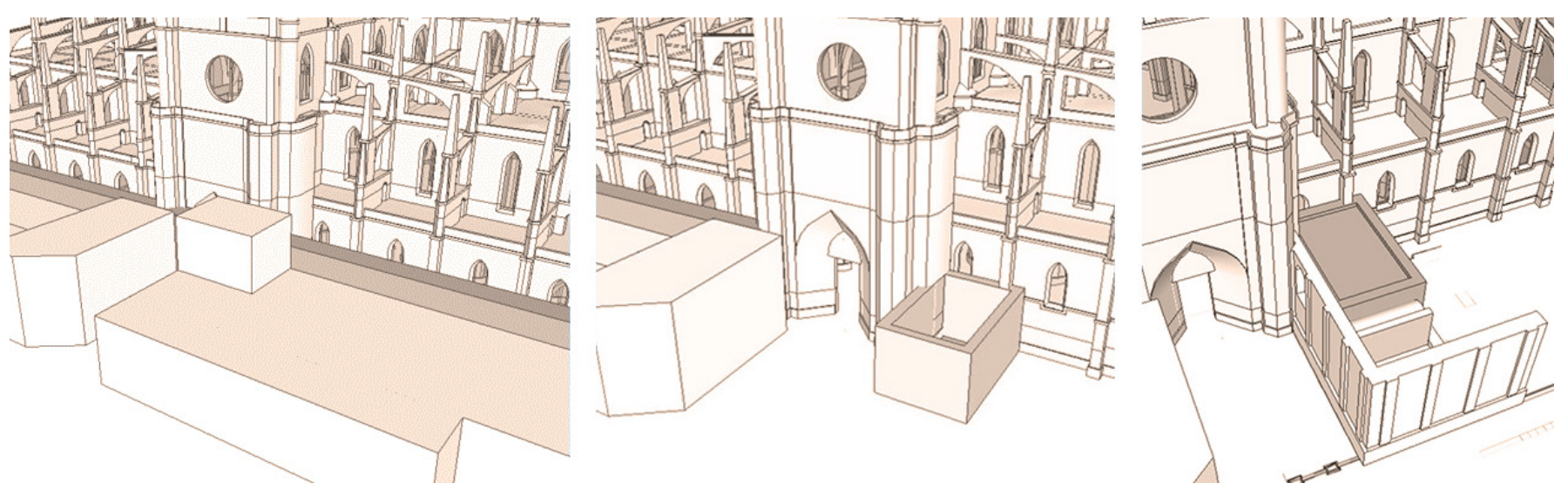

Figura 2. Tres etapas del proceso general. Dibujos de J. M. Guerrero 2013. 


\section{METODOLOGÍA}

La generación del modelo HBIM de la fachada se ha fundado sobre la base del conocimiento existente sobre la misma, en particular sobre un modelo conceptual previo que atañe a toda la catedral, mucho menos definido (Guerrero Vega 2010), y la lectura estratigráfica citada en la introducción, de la que se han extraído las claves para la caracterización de las unidades constructivas del modelo del fragmento seleccionado desde un nivel de detalle bajo a uno alto.

La lectura estratigráfica sobre la que se ha trabajado estaba integrada en una lectura documental y arquitectónica del edificio tratado (Fig. 3) (Pinto-Puerto 2013). Dado el carácter de este trabajo, se consideraron como unidades estratigráficas las regiones formadas por cada grupo de sillares que mantenían un aparejo semejante, y las discontinuidades que se producían entre ellos como fronteras. El cambio de material no se consideró como elemento diferenciador entre unidades, pues documentalmente se constató que fue coetáneo. Estas diferenciaciones se realizaron tanto por el exterior como por el interior del muro, verificando su relación con cada uno de los espacios contenidos y los acontecimientos sucedidos. A pesar de plantearse la metodología estratigráfica de forma simplificada, los resultados fueron suficientemente explícitos y útiles para plantear preguntas y obtener relaciones entre diversas fuentes documentales.

En paralelo a la revisión de la lectura estratigráfica, se realizó una captura digital fotogramétrica que ha proporcionado la información métrica necesaria para la generación del modelo tridimensional y ha permitido reconocer y delimitar cada unidad constructiva con mayor precisión. La nube de puntos de la captura digital se ha procesado en el software de ingeniería inversa Geomagic Design $X ®$, para la obtención y segmentación de una malla de triángulos a partir de la cual obtener las superficies simples y complejas que conforman el límite exterior del muro. Ese mismo entorno de trabajo ha servido para la resolución de las uniones entre dichas superficies y la obtención de una única superficie compuesta constituyente de la "cáscara" exterior del muro (Fig. 4). Esa superficie de alta precisión (en términos de adaptación a la captura digital fotogramétrica), combinada con otras superficies de menor precisión (basadas en levantamientos gráficos preexistentes), procesadas ya en un entorno de CAD, se constituye como el "molde" necesario para generar las entidades volumétricas, de tipo sólido, que posteriormente han sido categorizadas en el entorno de
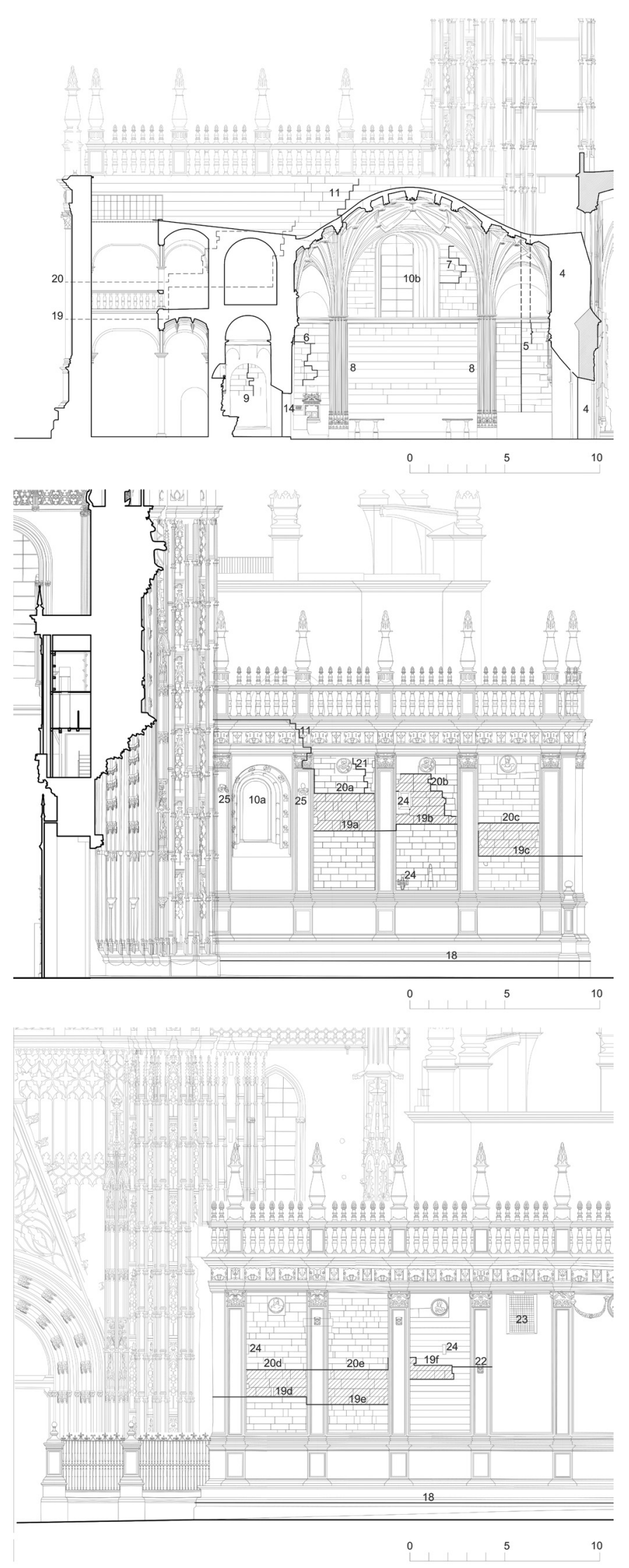

Figura 3. Sección y alzados con secuencia estratigráfica. Dibujos de F. Pinto-Puerto 2013. 

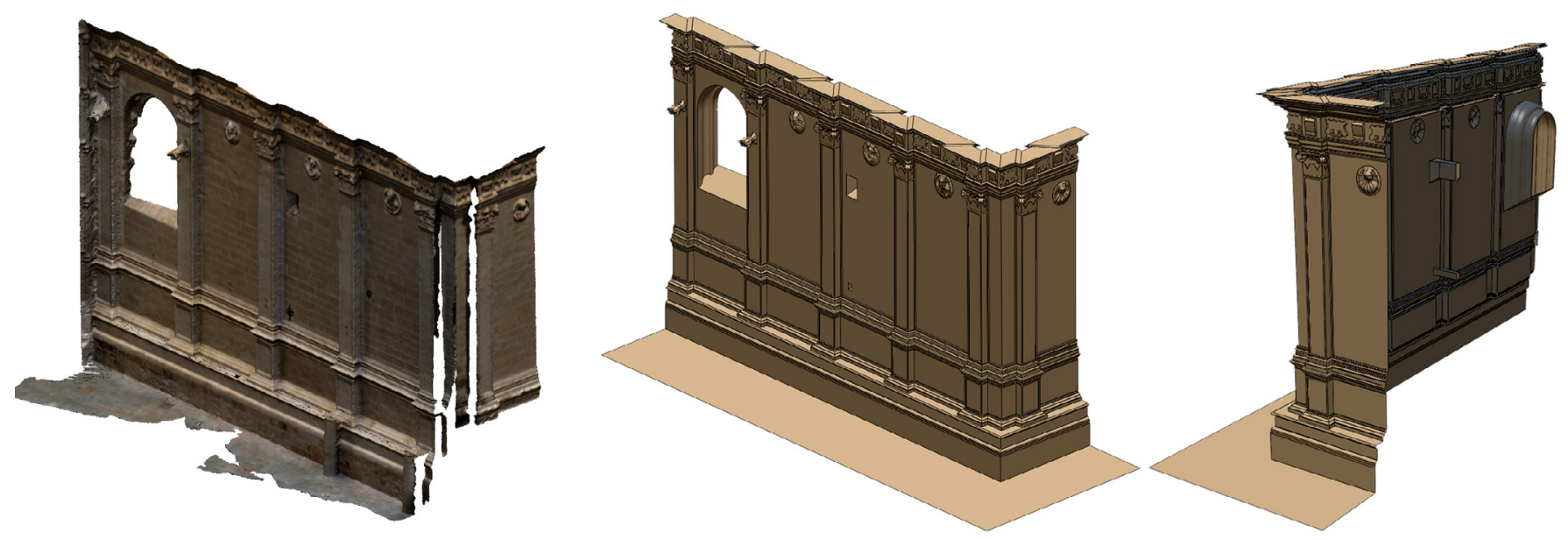

Figura 4. Vista axonométrica de la superficie mallada obtenida a partir de la CMD (izquierda). Vistas exterior e interior del modelo vectorial CAD una vez editado (centro y derecha).

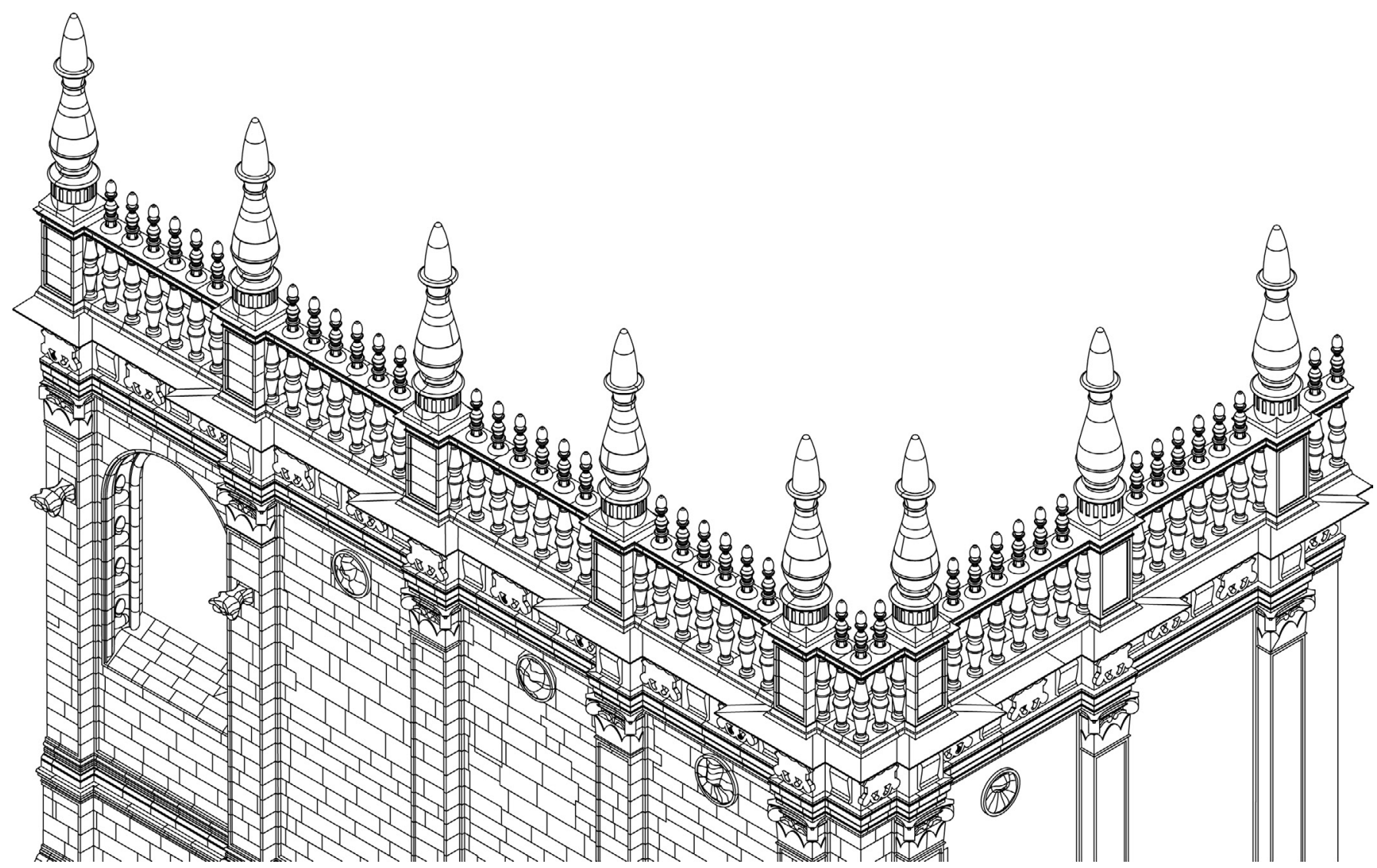

Figura 5. Proceso de edición en CAD. Sólido despiezado en entidades sólidas tridimensionales.

modelado BIM. Este último proceso se ha realizado en un software $\mathrm{CAD}$ convencional, en concreto Autodesk ${ }^{\circledR}$ AutoCAD ${ }^{\circledR}$. Este proceso de modelado se puede categorizar como "no paramétrico semiautomático" según la clasificación definida en Angulo-Fornos y Castellano-Román (2020: 6).
En este punto, el volumen sólido obtenido es único. Para poder avanzar en la caracterización constructiva pormenorizada, ha sido necesario dividir el sólido en piezas que se correspondían con las unidades constructivas básicas. Dadas las características singulares del caso de estudio se ha llevado la situación al extremo, de for- 
ma que esta unidad básica ha sido el sillar. Utilizando las ortofotografías derivadas de la fotogrametría se ha realizado una división sistemática del volumen sólido, obteniendo cada uno de los sillares de la fábrica (Fig. 5).

Posteriormente, se ha generado el modelo HBIM, importando el modelo vectorial, georreferenciándolo y poniéndolo en relación con el modelo genérico de la catedral antes citado y estableciendo los sistemas de referencias y el repertorio terminológico propios del elemento modelado. En este caso, el software utilizado ha sido Autodesk ${ }^{\circledR}$ Revit $\AA$.

Así, las entidades CAD se han ido importando a la plataforma BIM, asignándoles la categoría correspondiente. En este caso, se han importado dentro de un ejemplar de familia de categoría "muro", modelado como componente in situ. Este ejemplar de muro forma parte del proyecto de Revit ${ }^{\circledR}$ que, adecuadamente georreferenciado, compondrá, junto con otros modelos parciales, el modelo federado del templo.

No se disponía de registros o documentos suficientes como para deducir con precisión el espesor de las distintas hojas que componen la estructura de la fachada y la existencia de piezas dispuestas a modo de llaves que, atravesando la supuesta hoja interior de calicastrado, unen las hojas exteriores de piedra. La falta de certeza en este tipo de cuestiones, tanto geométricas o formales como de información asociada, no ha impedido la posibilidad de modelar las entidades en base a hipótesis bien fundadas y asignarles valores relativos a diversos niveles de conocimiento - $\mathrm{LOK}$ - a través de parámetros creados ex profeso en el modelo HBIM, en espera de tener información certera que permita su actualización. Dichos parámetros permiten la generación de imágenes gráficas tematizadas, en cualquier tipo de proyección, basadas en el LOK pormenorizado a la unidad básica de modelado. Con este mismo criterio, para entender de forma adecuada la configuración de esta fachada, y facilitar su interpretación, se han considerado también los espacios que encierra, básicamente los conjuntos conocidos como la sacristía de los Cálices y el Patio de los Oleos, modelados de forma más simplificada, adaptados a un nivel de conocimiento inferior.

\section{RESULTADOS}

La composición del ejemplar resultante se basa en la superposición de los distintos sólidos importados por separado en función de su nivel de detalle geométrico o formal, lo que
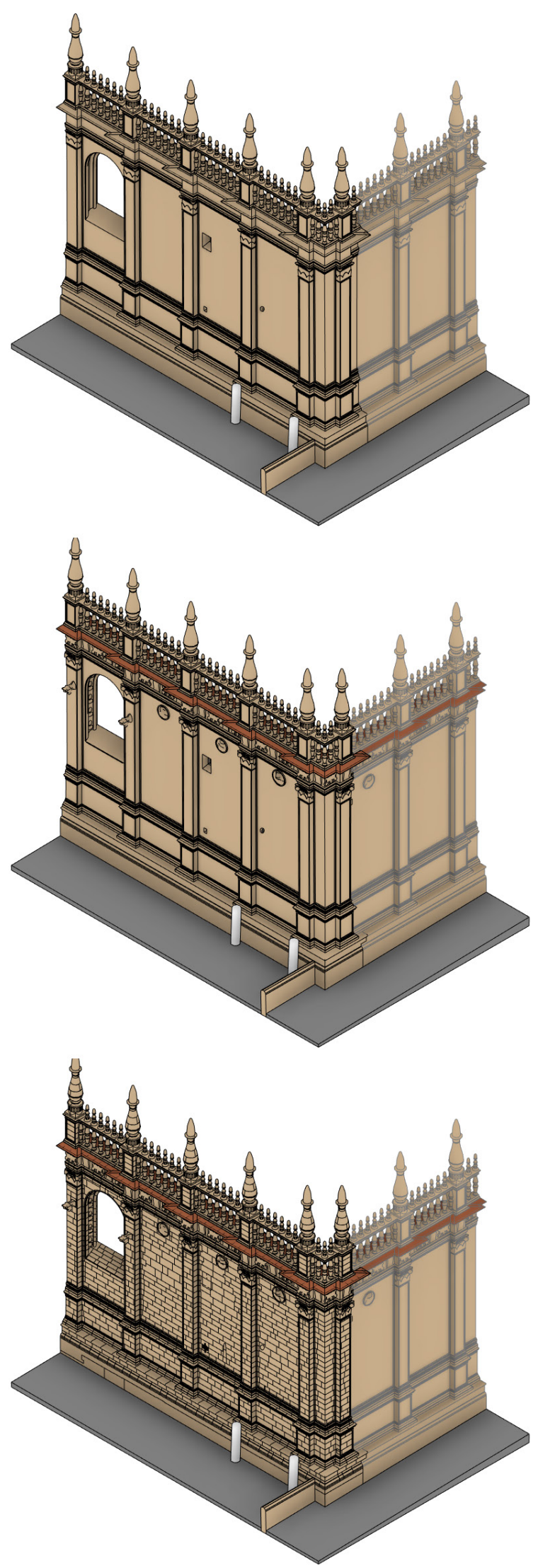

Figura 6. a) Nivel de detalle bajo. b) Nivel de detalle medio. c) Nivel de detalle alto. 
permite, utilizando un solo objeto, generar distintas visualizaciones dirigidas a volcados gráficos de distinta escala de representación, lo que implica además distintas posibilidades en cuanto a la cantidad o profundidad de la información introducida. De esta manera, cada sólido importado se convierte en una pieza asociada a uno de los tres niveles de detalle: bajo, medio y alto.

El Nivel de Detalle Bajo presenta el sólido completo del fragmento de muro sin elementos figurativos. Permite la producción de documentación gráfica a escala de representación asimilable a un anteproyecto de arquitectura de nueva planta $-1 / 200$ a $1 / 100-$. Reproduce fielmente la configuración geométrica y formal del elemento, resultado del proceso exhaustivo de modelado descrito con anterioridad. Una vista en sección de este elemento se presenta como una única entidad masiva, sin discriminación de sus capas materiales y despieces reales (Fig. 6a).

El Nivel de Detalle Medio presenta el sólido completo del fragmento con inclusión de elementos figurativos. Responde a las mismas características del nivel anterior, con aportación de mayor información a nivel gráfico. Permite obtener documentación gráfica a escala de representación asimilable a un proyecto básico de arquitectura de nueva planta (Fig. 6b).

El Nivel de Detalle Alto presenta el conjunto de sólidos resultado del despiece final desarrollado en el proceso de modelado. Permite la obtención de documentación gráfica a escala de representación asimilable a un proyecto de ejecución de arquitectura de nueva planta alcanzando desde proyecciones generales del objeto hasta detalles constructivos. Además de reproducir fielmente la configuración geométrica y formal del elemento, incluye la representación de los despieces reales correspondientes a los distintos estratos que conforman el muro (Fig. 6c).

A cada una de las piezas del modelo se le han asociado campos de información que las identifican y sitúan en el tiempo en relación con las demás. Entre estos campos de información se encuentran los datos relacionados con la secuencia estratigráfica. La introducción efectiva de los distintos conceptos de la estructura de información en el modelo se ha llevado a cabo mediante la creación de parámetros personalizados ligados a los mismos dentro del software BIM. Dichos parámetros se han incluido dentro de una lista llamada de "parámetros compartidos" registrada en un archivo de texto susceptible de ser vinculable a cualquier otro proyecto parcial o federado. Se han asignado los parámetros requeridos en base a tres niveles de desarrollo contenidos en el modelo HBIM, dos basados en la entidad sólida completa en dos niveles de detalle-diferenciados de cara a variaciones de escala en representaciones gráficas- y otro a partir de las entidades despiezadas. Estos niveles de desarrollo pueden considerarse, a los efectos del uso patrimonial del BIM, como Niveles de Conocimiento LOK.

De esta forma, a la entidad constituida por modelos volumétricos completos -ejemplar de categoría murose han asociado parámetros que se refieren al elemento como conjunto: identificación, información patrimonial básica sobre la adscripción estilística y autoría principal, figuras de protección jurídica que le afectan, registro de intervenciones y acciones de difusión relacionadas. Independientemente de su detalle gráfico - que ha llegado a ser de nivel alto por necesidades de representación-, podemos considerar que se adscribe a un nivel de conocimiento LOK300.

En el caso del modelo despiezado de la fachada, cada pieza es un objeto de registro y, por lo tanto, pueden asociarse parámetros relacionados con un mayor nivel de conocimiento, el requerido en este caso para gestionar la conservación preventiva del elemento. Así, a cada pieza se le asigna un identificador único y es caracterizada con parámetros de identificación, cronología, características litológicas, lesiones, tratamientos y acciones como la sustitución, que genera una cronología específica. Podemos considerar, por tanto, que se adscribe a un nivel de conocimiento LOK500.

Si los valores definidos en los párrafos anteriores se refieren a un LOK por elemento, siguiendo el criterio adoptado por el grupo de trabajo, es preceptivo asignar un LOK global tanto a los modelos parciales como al modelo federado. En este caso, el nivel de conocimiento asignado al modelo parcial que contiene el fragmento de fachada es LOK200 en base al estado de desarrollo actual. En el caso del modelo federado del templo, dado que el nivel actual de desarrollo consiste en un modelo volumétrico básico con la única inserción de un modelo parcial, podemos adscribirlo a un LOK100.

Las propiedades de gestión conferidas de forma automática a cada elemento, así como la asignación de los parámetros a las distintas entidades que conforman el modelo en función de su categoría y del sistema de relaciones implementado, supone el salto cualitativo desde un simple modelo gráfico tridimensional -desarrollado en cualquier software de dibujo digital o CAD- a un verdadero modelo de información -BIM o HBIM en este caso- capaz de ofrecer todas las ventajas de una base de 


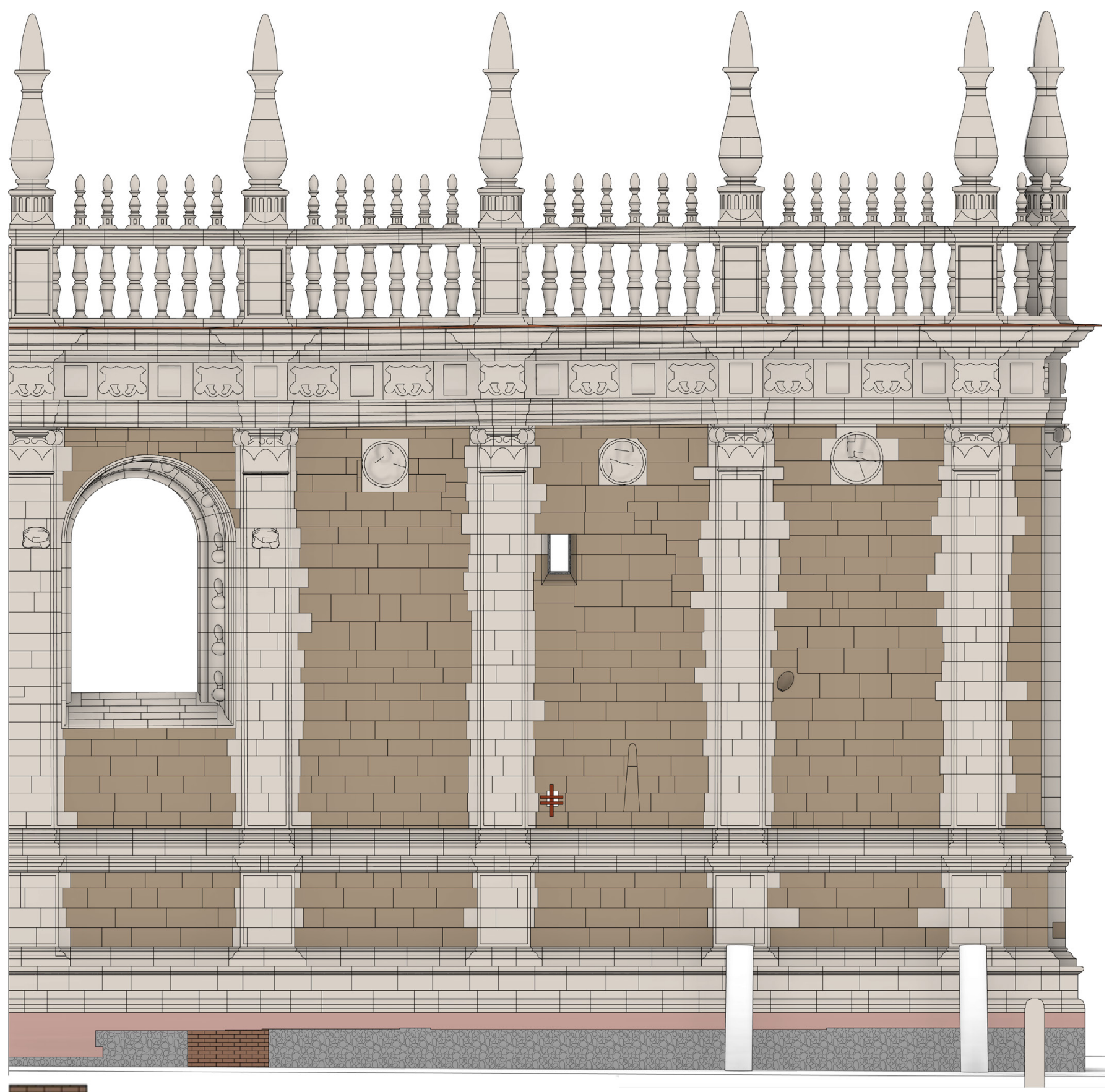

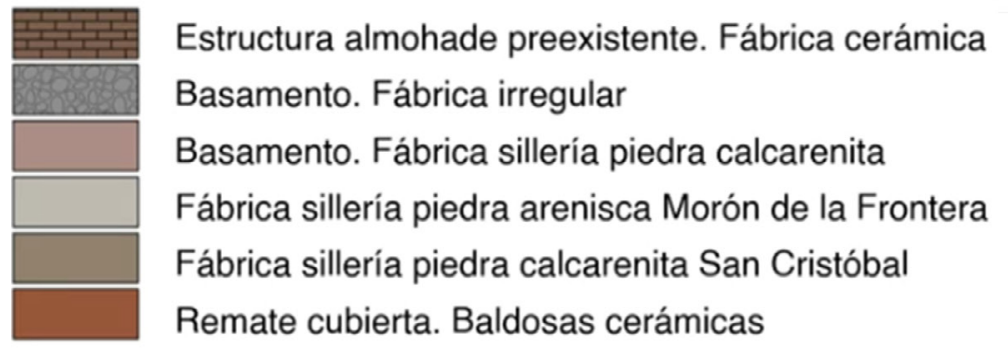

Figura 7. Vista en alzado del modelo filtrado por el parámetro "material". 

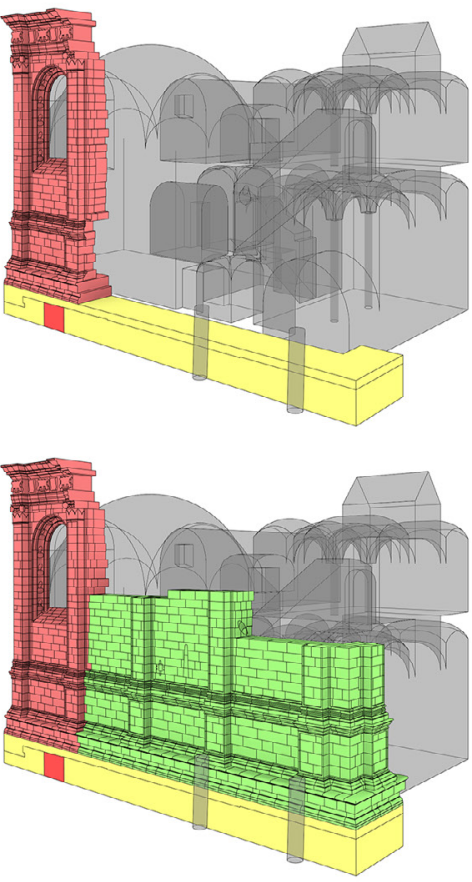

Estructura al

Nivelación indeterminada y amortización estructuras previas. Diego de Riaño Obra inicial de la capilla. Adosamiento a estribo tardogótico. Diego de Riaño Muro envolvente hasta primer nivel patio óleos. Creación del orden por Riaño (I) Muro envolvente hasta primer nivel patio óleos. Creación del orden por Riaño (II) Recrecido muro envolvente hasta cornisa Elementos ornamenta Pes Riano-Gainza (II) Recrecidomure

Terminaciones. Solado cubierta y balaustradas. Gainza Reformas contemporáneas

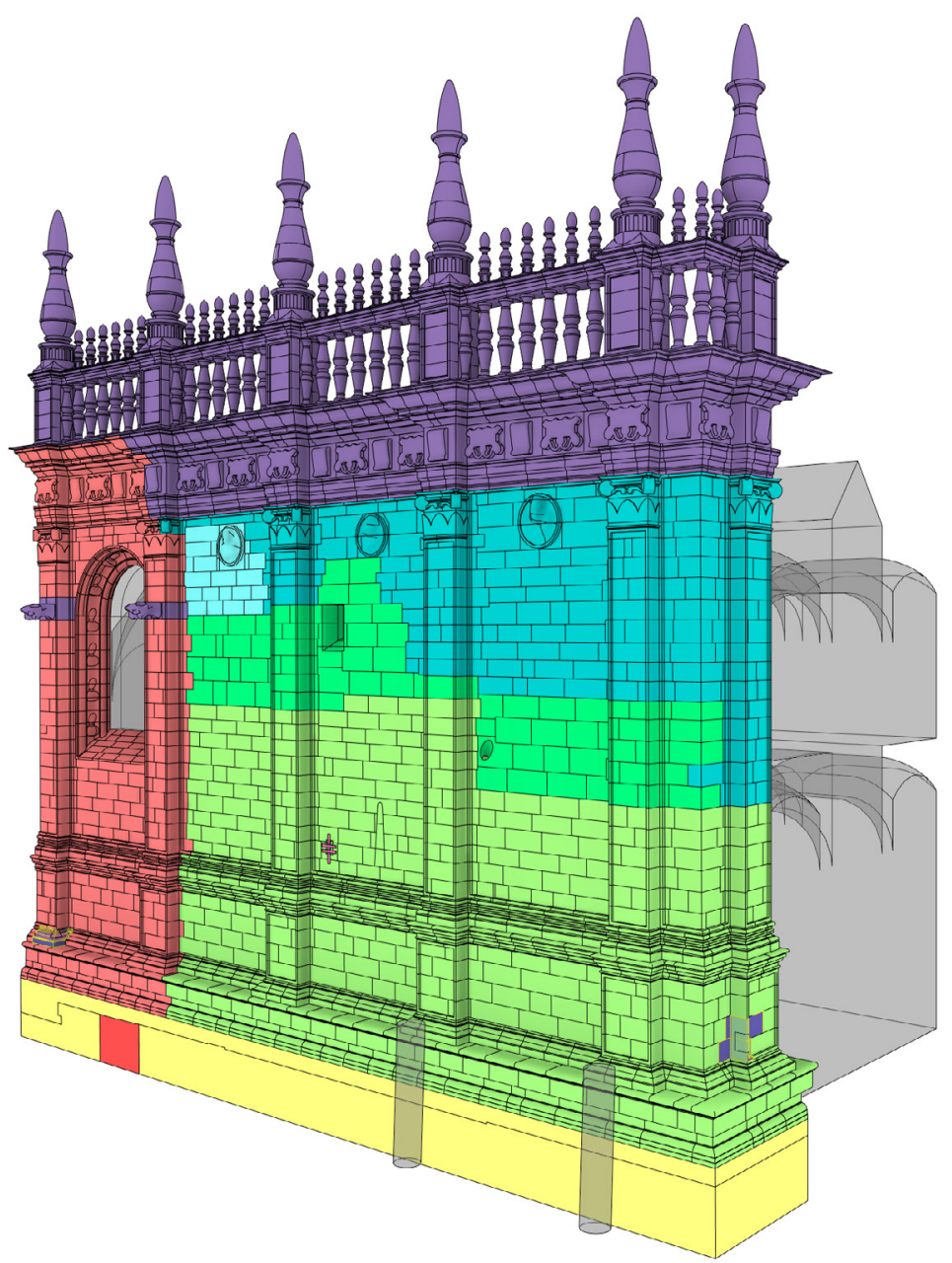

Figura 8. Perspectivas del modelo filtradas por el parámetro "ID Fase" que gestiona la información relativa a la estratigrafía del objeto de estudio.

datos gráfica. Así, una vez asignados los valores correspondientes dentro de cada parámetro a cada entidad, a partir de la información previamente sistematizada, estamos en disposición de poder solicitar al sistema consultas, filtrados de información o búsquedas condicionadas, en forma de gráficos tematizados o listados alfanuméricos, que nos ofrezcan nuevos puntos de partida a la hora de afrontar diversos tipos de análisis; o información actualizada en tiempo real sobre determinados aspectos de la realidad patrimonial del bien (Fig. 7).

Por otro lado, en el entorno BIM, los filtrados gráficos de información o consultas gráficas tematizadas pueden ser personalizados en combinación con herramientas de configuración de las vistas en las cuales son representadas. De esta manera, la visualización intencionada desde determinados puntos de vista -si consideramos perspectivas axonométricas o cónicas-o utilizando proyecciones diédricas, permiten la contemplación de las relaciones entre elementos y su información asociada desde nuevos enfoques difíciles de alcanzar sin contar con esta herramienta. En ese sentido, la implementación en el modelo del parámetro "ID Fase" ha permitido la incorporación de la información relativa a la estratigrafía, de manera que a cada unidad básica de modelado se le ha asignado el valor correspondiente a la unidad estratigráfica a la que pertenece (Fig. 8). De esta forma, es posible generar imágenes gráficas capaces de mostrar de forma paralela la evolución del fragmento estudiado y la configuración espacial del interior del cuadrante renacentista.

\section{CONCLUSIONES}

Se ha elaborado un modelo de información patrimonial HBIM de la fachada del cuadrante renacentista de la catedral de Sevilla con la capacidad de caracterizar la 
evolución constructiva de un elemento arquitectónico complejo, incorporando la lectura estratigráfica tridimensional de los paramentos.

El modelo gráfico se basa en una captura métrica digital fotogramétrica que es analizada e interpretada con las claves metodológicas del levantamiento arquitectónico. El modelo surge, en consecuencia, de una propuesta de interpretación de los datos métricos fundada sobre el conocimiento arqueológico y arquitectónico del elemento estudiado, diferenciando aquellos elementos cuya definición surge de un conocimiento cierto, de aquellos modelados según hipótesis pendientes de confirmación.

Se establecen tres niveles de desarrollo del modelo del elemento muro, como evolución de un primer nivel conceptual de la catedral, con el que estaría federado: dos que, diferenciados en dos niveles de detalle a nivel formal, abarcan todo el elemento estudiado en una única entidad y otro, derivado de un análisis arquitectónico y arqueológico existente, donde se ha delimitado cada unidad constructiva del conjunto. Los modelos de entidad única incorporan los parámetros de información relativos al conjunto del elemento. Esta información se completa con los parámetros de cada pieza del modelo despiezado, donde se incorporan los parámetros asociados a las acciones de conservación preventiva, permitiendo numerosas visualizaciones que mejoran la comprensión de la complejidad espacial y constructiva, $\mathrm{y}$, por otro lado, quedan registrada la información asociada al modelo.

\section{AGRADECIMIENTOS}

Los autores agradecen la colaboración del cabildo de la catedral de Sevilla y de la empresa Artyco S.L. por los medios puestos para el registro pormenorizado de la fachada durante las últimas obras de intervención.

\section{BIBLIOGRAFÍA}

Angulo-Fornos, R. y Castellano-Román, M. 2020: “HBIM as Support of Preventive Conservation Actions in Heritage Architecture. Experience of the Renaissance Quadrant Façade of the Cathedral of Seville", Applied Sciences, 10 (7), 2428. https://doi.org/10.3390/app10072428

Arévalo-Rodríguez, F. 2011: "El amurallamiento externo en la mezquita aljama de la Sevilla almohade: una hipótesis a partir de una muralla fosilizada, de las excavaciones arqueológicas y del análisis gráfico del parcelario", en La catedral sin la catedral, pp. 7-56. Aula Hernán Ruiz, Catedral de Sevilla, Sevilla.

Castellano-Román, M. y Pinto-Puerto, F. 2019: "Dimensions and Levels of Knowledge in Heritage Building Information Modelling, HBIM: The model of the Charterhouse of Jerez (Cádiz, Spain)", Digital Applications in Archaeology and Cultural Heritage, 14, e00110. https://doi.org/10.1016/j. daach.2019.e00110

Guerrero Vega, J. M. 2010: "El informe de 1513 de Alonso Rodríguez", en A. Jiménez Martín (ed.), La catedral después de Carlín, pp. 31-74. Aula Hernán Ruiz, Catedral de Sevilla, Sevilla.

Pinto-Puerto, F. 2006: "Fábrica y forma del templo gótico", en A. Jiménez-Martín (coord.), La catedral gótica de Sevilla: Fundación y fábrica de la obra nueva, pp. 211-295. Universidad de Sevilla, Sevilla.

Pinto-Puerto, F. 2013: "La Sacristía de los Cálices. Aportaciones desde el análisis de sus fábricas y los sistemas de control formal", en F. Guerra-Librero Fernández y A. Jiménez Martín (eds.), La Catedral entre 1434 y 1517 historia y conservación, pp. 163-235. Aula Hernán Ruiz, Catedral de Sevilla, Sevilla.

Rodríguez Estévez, J. C. 1998: Los canteros de la Catedral de Sevilla: del Gótico al Renacimiento. Diputación de Sevilla, Sevilla.

Rua, H. y Gil, A. 2014: "Automation in heritage - Parametric and associative design strategies to model inaccessible monuments: The case-study of eighteenth-century Lisbon Águas Livres Aqueduct", Digital Applications in Archaeology and Cultural Heritage, 1 (3), pp. 82-91. https://doi. org/10.1016/j.daach.2014.06.002

Tabales-Rodríguez, M. Á. y Jiménez Sancho, Á. 2002: "La cilla de la catedral y el sector meridional de la mezquita aljama de Sevilla”, en Magna Hispalensis (I): Recuperación de la Aljama Almohade, pp. 229-296. Aula Hernán Ruiz, Cabildo Metropolitano, Sevilla 